\title{
On smoothers for multigrid of the second kind
}

\author{
Burak Aksoylu' ${ }^{1,2}$ | Adem Kaya ${ }^{3}$ (i)
}

${ }^{1}$ CCDC Army Research Laboratory, Aberdeen, Maryland

${ }^{2}$ Department of Mathematics, Wayne State University, Detroit, Michigan

${ }^{3}$ Department of Mathematics, Izmir Institute of Technology, Izmir, Turkey

\section{Correspondence}

Burak Aksoylu, CCDC Army Research Laboratory, Aberdeen Proving Ground, Aberdeen, MD 21005.

Email: burak@wayne.edu

\section{Funding information}

Army Research Laboratory, Grant/Award Number: W911NF-18-2-0090

\begin{abstract}
Summary
We study smoothers for the multigrid method of the second kind arising from Fredholm integral equations. Our model problems use nonlocal governing operators that enforce local boundary conditions. For discretization, we utilize the Nyström method with the trapezoidal rule. We find the eigenvalues of matrices associated to periodic, antiperiodic, and Dirichlet problems in terms of the nonlocality parameter and mesh size. Knowing explicitly the spectrum of the matrices enables us to analyze the behavior of smoothers. Although spectral analyses exist for finding effective smoothers for 1D elliptic model problems, to the best of our knowledge, a guiding spectral analysis is not available for smoothers of a multigrid of the second kind. We fill this gap in the literature. The Picard iteration has been the default smoother for a multigrid of the second kind. Jacobi-like methods have not been considered as viable options. We propose two strategies. The first one focuses on the most oscillatory mode and aims to damp it effectively. For this choice, we show that weighted-Jacobi relaxation is equivalent to the Picard iteration. The second strategy focuses on the set of oscillatory modes and aims to damp them as quickly as possible, simultaneously. Although the Picard iteration is an effective smoother for model nonlocal problems under consideration, we show that it is possible to find better than ones using the second strategy. We also shed some light on internal mechanism of the Picard iteration and provide an example where the Picard iteration cannot be used as a smoother.
\end{abstract}

\section{KEYWORDS}

local boundary condition, multigrid of the second kind, nonlocal operator, smoother, the Fredholm integral equation of the second kind, the Picard iteration

\section{1 | INTRODUCTION}

We study smoothers for solving systems arising from Fredholm integral equations of the second kind known as the multigrid of the second kind. The Picard iteration is a well known smoother for Fredholm second kind systems. ${ }^{1 \text { p.495, } 2}$ The multigrid method for such systems dates back to late $1970 \mathrm{~s}^{3-6}$ The Fredholm operators of second kind under consideration arise from nonlocal operators introduced in the works of Aksoylu et al.$^{7-9}$ The operators are inspired by peridynamics, a nonlocal formulation of continuum mechanics. ${ }^{10}$ Peridynamics is capable of quantitatively predicting the dynamics of propagating cracks, including bifurcation. Its effectiveness has been established in sophisticated applications such as Kalthoff-Winkler experiments of the fracture of a steel plate with notches, ${ }^{11,12}$ fracture and failure of composites, nanofiber networks, and polycrystal fracture. ${ }^{13-16}$ 
Unlike traditional nonlocal operators, our operators enforce local boundary conditions (BC). When these operators are extended to vector valued problems, we anticipate that our operators will help apply peridynamics to problems that require local BC. In addition, the ability to enforce local BC makes these operators attractive because our approach will avoid altogether the surface effects seen in peridynamics. In the work of Aksoylu et al., ${ }^{8}$ the theoretical foundations were laid, and in the work of Aksoylu et al., ${ }^{7}$ the foundations were applied to prominent BC such as Dirichlet and Neumann, and the numerical implementation of the corresponding wave propagation was presented. The novel operators were extended to higher dimensions. ${ }^{17}$ An overview of how to apply functional calculus to general nonlocal problems in a methodical way was provided. ${ }^{18}$ The conditioning and error analysis of the novel operators was provided. ${ }^{19}$

The convergence speed of a multigrid method critically depends on the smoother used. Effective smoother means that it enables a fast multigrid convergence and, hence, is vital from the linear system point of view. Jacobi methods have been frequently used probably due to their ease of use. Because the operators of the Fredholm second kind have a smoothing property, the Picard iteration has been the default smoother ${ }^{20}$ in multigrid methods. Jacobi-like smoothers have not been considered as viable options. Although spectral analyses exist for finding effective smoothers for 1D elliptic model problems, ${ }^{20-22}$ to the best of our knowledge, a guiding spectral analysis is not available for Fredholm second-type problems. We fill the gap in the literature by revealing the internal mechanism of the Picard iteration and show that Jacobi-like methods can be used as smoothers for multigrid methods.

We obtain the discrete systems by utilizing the Nyström method with the trapezoidal rule. ${ }^{19}$ We obtain the spectrum of system matrices of periodic, antiperiodic, and Dirichlet problems using eigenfunctions of the original continuous operators. Due to analytical construction, we have direct access to the explicit expression of the eigenvalues of the operators in terms of nonlocality parameter $\delta$. Using the eigenfunctions of the continuous operators, we find the eigenvalues of the matrices in terms of mesh size $h$ and $\delta$. The explicit expression of the spectra is instrumental in finding effective smoothers because direct spectral analysis of the weighted (damped)-Jacobi relaxation becomes possible.

For a relaxation scheme to be a smoother, it must damp the oscillatory modes effectively. Based on this fact, we propose two strategies to construct smoothers using weighted-Jacobi relaxation. The first strategy depends on the idea of damping the most oscillatory mode as quickly as possible. We find a parameter that accomplishes this task in the case of the antiperiodic problem. In fact, we show that the weighted-Jacobi becomes equivalent to the Picard iteration. Through this equivalence we shed some light on the working principle of the Picard iteration from the spectral point of view. The second strategy depends on the idea of damping the oscillatory modes as quickly as possible, simultaneously. The spectral analysis for the second strategy applied to 1D Poisson boundary value problem can be found in other works. ${ }^{21,22}$ Furthermore, a good reference of smoothing analysis for several other problems is in the work of Trottenberg et al. ${ }^{23} \mathrm{We}$ provide a way to numerically find an optimal parameter with given $\delta$ and $h$. A smoother is designed to produce a fast and scalable multigrid method. Based on this fact, we compare relaxation schemes obtained from the two strategies.

We collect the statements of our three main results in this study.

- Main Result 1: Identification of the internal mechanism of the Picard iteration. More precisely, the Picard iteration aims to damp the most oscillatory mode.

- Main Result 2: For Fredholm second kind problems, a smoother strategy based on simultaneous damping of the oscillatory modes is more effective than damping the most oscillatory mode.

- Main Result 3: The weighted-Jacobi iteration can be used as a smoother and with a suitable weight it is equivalent to the Picard iteration. In certain cases, the weighted-Jacobi iteration can even outperform the Picard iteration.

The remainder of this paper is structured as follows. In Section 2, we introduce the model problems and the nonlocal operators that enforce local BC. In Section 3, we present the spectrum of the underlying matrices explicitly. In Section 4, we construct the smoothers based on two strategies. We also provide the spectral analysis for their iteration matrices. In Section 5, we compare the two smoothing strategies. A discussion devoted to the Picard iteration and its effectiveness is presented in Section 6. We conclude in Section 7.

\section{2 | MODEL PROBLEMS}

Our model problems use nonlocal governing operators that enforce local periodic, antiperiodic, Neumann, and Dirichlet BC. We briefly explain their construction. We set the domain $\Omega:=[-1,1]$. For $x, x^{\prime} \in \Omega$, it follows that the convolution 
argument $x^{\prime}-x \in \widehat{\Omega}:=[-2,2]$. Hence, the kernel function $C(x)$ needs to be extended from $\Omega$ to the domain of $\widehat{C}\left(x^{\prime}-x\right)$, which is $\widehat{\Omega}$. Furthermore, $C(x)$ is assumed to be nonnegative and even. Namely,

$$
C(-x)=C(x)
$$

The important choice of $C(x)$ is the canonical kernel function $\chi_{\delta}(x)$ whose only role is the representation of the nonlocal neighborhood, called the horizon, by a characteristic function. More precisely, for $x \in \Omega$,

$$
\chi_{\delta}(x):= \begin{cases}1, & x \in(-\delta, \delta) \\ 0, & \text { otherwise }\end{cases}
$$

The size of nonlocality is determined by $\delta$ and we assume $0<\delta<1$.

In the construction of the novel operators, a crucial ingredient is first restricting $\widehat{C}$ to $\Omega$ and then suitably extending it back to $\widehat{\Omega}$. To this end, we define the periodic and antiperiodic extensions of $C(x)$ from $[-1,1]$ to $[-2,2]$, respectively, as follows:

$$
\widehat{C}_{\mathrm{p}}(x):=\left\{\begin{array}{ll}
C(x+2), & x \in[-2,-1), \\
C(x), & x \in[-1,1], \\
C(x-2), & x \in(1,2],
\end{array} \widehat{C}_{\mathrm{a}}(x):= \begin{cases}-C(x+2), & x \in[-2,-1), \\
C(x), & x \in[-1,1], \\
-C(x-2), & x \in(1,2] .\end{cases}\right.
$$

Throughout this paper, we assume that

$$
u, C \in L^{2}(\Omega) .
$$

Even and odd parts of a univariate function $u(x)$ are used in the governing operators. We utilize the orthogonal projections that give the even and odd parts, respectively, of a univariate function by $P_{e}, P_{o}: L^{2}(\Omega) \rightarrow L^{2}(\Omega)$, whose definitions are

$$
P_{e} u(x):=\frac{u(x)+u(-x)}{2}, \quad P_{o} u(x):=\frac{u(x)-u(-x)}{2} .
$$

In addition, we define

$$
\mathcal{T}_{\mathrm{p}} u(x):=\int_{\Omega} \widehat{C}_{\mathrm{p}}\left(x^{\prime}-x\right) u\left(x^{\prime}\right) d x^{\prime} \quad \text { and } \quad \mathcal{T}_{\mathrm{a}} u(x):=\int_{\Omega} \widehat{C}_{\mathrm{a}}\left(x^{\prime}-x\right) u\left(x^{\prime}\right) d x^{\prime}
$$

Let $c=\int_{\Omega} C(x) d x$. We define the novel operators enforce local periodic and antiperiodic BC, respectively:

$$
\mathcal{M}_{\mathrm{p}} u(x):=c u(x)-\mathcal{T}_{\mathrm{p}} u(x) \text { and } \mathcal{M}_{\mathrm{a}} u(x):=c u(x)-\mathcal{T}_{\mathrm{a}} u(x)
$$

In addition, we define the novel operators that enforce local Neumann and Dirichlet BC, respectively:

$$
\begin{aligned}
\mathcal{M}_{\mathbb{N}} u(x) & :=c u(x)-\int_{\Omega}\left[\widehat{C}_{\mathrm{p}}\left(x^{\prime}-x\right) P_{e} u\left(x^{\prime}\right)+\widehat{C}_{\mathrm{a}}\left(x^{\prime}-x\right) P_{o} u\left(x^{\prime}\right)\right] d x^{\prime} \\
& =: \mathcal{T}_{\mathrm{N}} u(x) \\
\mathcal{M}_{\mathrm{D}} u(x) & :=c u(x)-\int_{\Omega}\left[\widehat{C}_{\mathrm{a}}\left(x^{\prime}-x\right) P_{e} u\left(x^{\prime}\right)+\widehat{C}_{\mathrm{p}}\left(x^{\prime}-x\right) P_{o} u\left(x^{\prime}\right)\right] d x^{\prime} \\
& =: \mathcal{T}_{\mathrm{D}} u(x) .
\end{aligned}
$$

The operators $\mathcal{T}_{\mathrm{BC}}, \mathrm{BC}=\{\mathrm{p}, \mathrm{a}, \mathrm{N}, \mathrm{D}\}$ are self-adjoint and compact. We are primarily interested in the choice of $C(x)=\chi_{\delta}(x)$. This choice is the most representative among kernel functions because it represents the source of nonlocality, that is, the horizon. 
One can easily identify the associated kernels with the operators. By writing $P_{e}$ and $P_{o}$ explicitly and utilizing a simple change of variables, the operators can be written as

$$
\begin{aligned}
& \mathcal{M}_{\mathrm{D}} u(x)=c u(x)-\int_{\Omega} K_{\mathrm{D}}\left(x, x^{\prime}\right) u\left(x^{\prime}\right) d x^{\prime}, \\
& \mathcal{M}_{\mathrm{N}} u(x)=c u(x)-\int_{\Omega} K_{\mathrm{N}}\left(x, x^{\prime}\right) u\left(x^{\prime}\right) d x^{\prime},
\end{aligned}
$$

where

$$
\begin{aligned}
& K_{\mathrm{D}}\left(x, x^{\prime}\right):=\frac{1}{2}\left\{\left[\widehat{C}_{\mathrm{a}}\left(x^{\prime}-x\right)+\widehat{C}_{\mathrm{a}}\left(x^{\prime}+x\right)\right]+\left[\widehat{C}_{\mathrm{p}}\left(x^{\prime}-x\right)-\widehat{C}_{\mathrm{p}}\left(x^{\prime}+x\right)\right]\right\}, \\
& K_{\mathrm{N}}\left(x, x^{\prime}\right):=\frac{1}{2}\left\{\left[\widehat{C}_{\mathrm{p}}\left(x^{\prime}-x\right)+\widehat{C}_{\mathrm{p}}\left(x^{\prime}+x\right)\right]+\left[\widehat{C}_{\mathrm{a}}\left(x^{\prime}-x\right)-\widehat{C}_{\mathrm{a}}\left(x^{\prime}+x\right)\right]\right\} .
\end{aligned}
$$

Utilizing the explicit expressions of the kernel functions in (2) and (3), we show how the operators enforce homogeneous Dirichlet and Neumann BC. By the Lebesgue dominated convergence theorem, the limit in the definition of the Dirichlet $\mathrm{BC}$ can be interchanged with the integral. We check the boundary values by plugging $x= \pm 1$ in (2).

$$
\mathcal{M}_{\mathrm{D}} u( \pm 1)=c u( \pm 1)-\int_{\Omega} K_{\mathrm{D}}\left( \pm 1, x^{\prime}\right) u\left(x^{\prime}\right) d x^{\prime}
$$

The functions $\widehat{C}_{\mathrm{p}}$ and $\widehat{C}_{\mathrm{a}}$ are 2-periodic and 2-antiperiodic, respectively, therefore, we have

$$
\widehat{C}_{\mathrm{p}}\left(x^{\prime} \mp 1\right)=\widehat{C}_{\mathrm{p}}\left(x^{\prime} \pm 1\right) \text { and } \widehat{C}_{\mathrm{a}}\left(x^{\prime} \mp 1\right)=-\widehat{C}_{\mathrm{a}}\left(x^{\prime} \pm 1\right) .
$$

Hence, the integrand in (1) vanishes, that is, $K_{\mathrm{D}}\left( \pm 1, x^{\prime}\right)=0$. Therefore, we arrive at

$$
\mathcal{M}_{\mathrm{D}} u( \pm 1)=c u( \pm 1)
$$

When we assume that $u$ satisfies homogeneous Dirichlet $\mathrm{BC}$, that is, $u( \pm 1)=0$, we conclude that the operator $\mathcal{M}_{\mathrm{D}}$ enforces homogeneous Dirichlet $\mathrm{BC}$ as well. The case of Neumann $\mathrm{BC}$ follows from a similar line of argument involving differentiation at $x= \pm 1$; see Section 3.2 in the work of Aksoylu et al. ${ }^{17}$

In order to avoid integration over the jumps of kernel functions, we prefer to utilize the following representation of the operators, which leads to a more convenient implementation:

$$
\begin{gathered}
\mathcal{T}_{\mathrm{p}} u(x):= \begin{cases}\int_{-1}^{x+\delta} u\left(x^{\prime}\right) d x^{\prime}+\int_{x+2-\delta}^{1} u\left(x^{\prime}\right) d x^{\prime}, & x \in[-1,-1+\delta), \\
\int_{x-\delta}^{x+\delta} u\left(x^{\prime}\right) d x^{\prime}, & x \in[-1+\delta, 1-\delta], \\
\int_{-1}^{x-2+\delta} u\left(x^{\prime}\right) d x^{\prime}+\int_{x-\delta}^{1} u\left(x^{\prime}\right) d x^{\prime}, & x \in(1-\delta, 1],\end{cases} \\
\mathcal{T}_{\mathrm{a}} u(x):= \begin{cases}\int_{-1}^{x+\delta} u\left(x^{\prime}\right) d x^{\prime}-\int_{x+2-\delta}^{1} u\left(x^{\prime}\right) d x^{\prime}, & x \in[-1,-1+\delta), \\
\int_{x-\delta}^{x+\delta} u\left(x^{\prime}\right) d x^{\prime}, & x \in[-1+\delta, 1-\delta], \\
-\int_{-1}^{x-2+\delta} u\left(x^{\prime}\right) d x^{\prime}+\int_{x-\delta}^{1} u\left(x^{\prime}\right) d x^{\prime}, & x \in(1-\delta, 1],\end{cases} \\
\mathcal{T}_{\mathrm{N}} u(x):= \begin{cases}\int_{-1}^{x+\delta} u\left(x^{\prime}\right) d x^{\prime}+\int_{-1}^{-x-2+\delta} u\left(x^{\prime}\right) d x^{\prime}, & x \in[-1,-1+\delta), \\
\int_{x-\delta}^{x+\delta} u\left(x^{\prime}\right) d x^{\prime}, & x \in[-1+\delta, 1-\delta], \\
\int_{-x+2-\delta}^{1} u\left(x^{\prime}\right) d x^{\prime}+\int_{x-\delta}^{1} u\left(x^{\prime}\right) d x^{\prime}, & x \in(1-\delta, 1],\end{cases} \\
\mathcal{T}_{\mathrm{D}} u(x):= \begin{cases}\int_{-1}^{x+\delta} u\left(x^{\prime}\right) d x^{\prime}-\int_{-1}^{-x-2+\delta} u\left(x^{\prime}\right) d x^{\prime}, & x \in[-1,-1+\delta), \\
\int_{x-\delta}^{x+\delta} u\left(x^{\prime}\right) d x^{\prime}, & x \in[-1+\delta, 1-\delta], \\
-\int_{-x+2-\delta}^{1} u\left(x^{\prime}\right) d x^{\prime}+\int_{x-\delta}^{1} u\left(x^{\prime}\right) d x^{\prime}, & x \in(1-\delta, 1] .\end{cases}
\end{gathered}
$$


For discretization, we utilize the Nyström method with the trapezoidal rule with uniformly distributed $N$ nodes where $N$ is odd. One can use other discretization rules, but we find the trapezoidal rule more useful because it allows us to write the expression of eigenvalues in a convenient way. We obtain the following system matrices:

$$
K_{\mathrm{BC}} \mathbf{u}_{\mathrm{BC}}=\mathbf{f}_{\mathrm{BC}}
$$

The system matrices $K_{\mathrm{BC}}$ are not symmetric. With simple algebraic manipulations, it is possible to obtain symmetric matrices $A_{\mathrm{BC}}$.

For the periodic problem, using the BC, we set $\left(\mathbf{u}_{\mathrm{p}}\right)_{1}=\left(\mathbf{u}_{\mathrm{p}}\right)_{N}$ and $\left(\mathbf{f}_{\mathrm{p}}\right)_{1}=\left(\mathbf{f}_{\mathrm{p}}\right)_{N}$. When we add the last column to the first one, we see that the first and last rows are identical. Using $\left(\mathbf{f}_{\mathrm{p}}\right)_{1}=\left(\mathbf{f}_{\mathrm{p}}\right)_{N}$, we can eliminate the last row because it is identical to the first row. Similarly, for the antiperiodic problem, we set $\left(\mathbf{u}_{\mathrm{a}}\right)_{1}=-\left(\mathbf{u}_{\mathrm{a}}\right)_{N}$ and $\left(\mathbf{f}_{\mathrm{a}}\right)_{1}=-\left(\mathbf{f}_{\mathrm{a}}\right)_{N}$. When we subtract the last column from the first one, we see that the first and last rows are identical. Using $\left(\mathbf{f}_{a}\right)_{1}=-\left(\mathbf{f}_{a}\right)_{N}$, we can eliminate the last row.

For the Neumann problem, we multiply the first and last rows by $1 / 2$. The entries $\left(\mathbf{f}_{\mathrm{N}}\right)_{1}$ and $\left(\mathbf{f}_{\mathrm{N}}\right)_{N}$ are also multiplied by $1 / 2$. This multiplication operation gives an equivalent system matrix, which is symmetric. For the Dirichlet problem, the values $\left(\mathbf{u}_{\mathrm{D}}\right)_{1}$ and $\left(\mathbf{u}_{\mathrm{D}}\right)_{N}$ are known because they are part of the BC. By deleting the first and last columns and the first and last rows, we obtain a symmetric system matrix. For the structure of the matrices and further details, see the work of Aksoylu et al. ${ }^{19}$ We note that the matrices $A_{\mathrm{BC}}, \mathrm{BC}=\{\mathrm{p}, \mathrm{a}\}$ are of size $(N-1) \times(N-1)$, whereas the matrices $A_{\mathrm{N}}$ and $A_{\mathrm{D}}$ are of size $N \times N$ and $N-2 \times N-2$, respectively. In our discretization, the ratio $R=\delta / h \geq 1$ always corresponds to a positive integer.

\section{3 | DERIVATION OF EIGENVALUES OF SYSTEM MATRICES}

We calculate the eigenvalues of the system matrices $A_{\mathrm{BC}}, \mathrm{BC}=\{\mathrm{p}, \mathrm{a}, \mathrm{D}\}$. We know that the operators $\mathcal{M}_{\mathrm{BC}}, \mathrm{BC}=\{\mathrm{p}, \mathrm{N}\}$ contain zero as an eigenvalue and, hence, are not invertible. Similarly, their corresponding system matrices $A_{\mathrm{BC}}, \mathrm{BC}=$ $\{\mathrm{p}, \mathrm{N}\}$ contain zero eigenvalue and they are not invertible either. Therefore, we will not consider them directly when we study smoothers. However, because the matrix $A_{\mathrm{D}}$ assumes eigenvalues of the matrix $A_{\mathrm{p}},{ }^{19}$, Lemma 4.2 we need to derive the spectrum of $A_{\mathrm{p}}$.

\section{1 | Periodic matrix $A_{\mathrm{p}}$}

The matrix $A_{\mathrm{p}}$ is symmetric positive semidefinite and Toeplitz. It also satisfies the zero row sum property and, hence, has a zero eigenvalue. We present its eigenpairs.

Lemma 1. Let $\mathbf{v}_{k}^{i}$ denote the ith component of the vector $\mathbf{v}_{k}$. Then, the matrix $A_{\mathrm{p}}$ assumes the eigenvectors, for $1 \leq i \leq$ $N-1$,

$$
\mathbf{v}_{k}^{i}:= \begin{cases}\cos \left(\frac{(i-1)(k-1) 2 \pi}{N-1}\right), & 1 \leq k \leq \frac{N+1}{2}, \\ \sin \left(\frac{(i-1)(k-1) 2 \pi}{N-1}\right), & \frac{N+1}{2}<k \leq N-1,\end{cases}
$$

with the corresponding eigenvalues where $h:=\frac{2}{N-1}$,

$$
\begin{aligned}
\lambda_{k}\left(A_{\mathrm{p}}\right): & =2 \delta+h \cos (\delta(k-1) \pi)+h-2 h \sum_{l=0}^{R} \cos (l(k-1) \pi h) \\
& = \begin{cases}0, & k=1, \\
2 \delta-h \frac{\sin ((k-1) \pi \delta) \sin ((k-1) \pi h)}{2 \sin ^{2}\left(\frac{(k-1) \pi h}{2}\right)}, & k=2, \ldots, N-1 .\end{cases}
\end{aligned}
$$


The eigenvalues have the property, for $k=1, \ldots,(N-1) / 2$,

$$
\lambda_{k}\left(A_{\mathrm{p}}\right)=\lambda_{N-k}\left(A_{\mathrm{p}}\right)
$$

Hence, the eigenvalues have geometric multiplicity 2 except $\lambda_{(N-1) / 2} A_{\mathrm{p}}$ whose geometric multiplicity is 1 .

Proof. Note that the vectors in (7) are orthogonal:

$$
\mathbf{v}_{k} \cdot \mathbf{v}_{l}:= \begin{cases}0, & 1 \leq k \neq l \leq N-1 \\ \frac{N-1}{2}, & 2 \leq k=l \leq N-1 \\ N-1, & k=l=1\end{cases}
$$

For computational convenience, we define the shifted matrix

$$
B_{\mathrm{p}}:=2 \delta I-A_{\mathrm{p}}
$$

and consider its eigenvalues. Let $\mathbf{b}_{i}$ denote the $i$ th row of $B_{\mathrm{p}}$ with $i=1, \ldots, N-1$. For $k=1, \ldots, N-1$, we want to show that $\mathbf{b}_{i} \cdot \mathbf{v}_{k}=\lambda_{k}\left(B_{\mathrm{p}}\right) \mathbf{v}_{k}^{i}$.

Depending on which interval $x$ comes from in (4), the rows originate from three different integral equations. The rows $\mathbf{b}_{i}, R<i<N-R$ are less involved because they are obtained from the discretization of the operator

$$
\int_{x-\delta}^{x+\delta} u\left(x^{\prime}\right) d x^{\prime}=f, \quad x \in[-1+\delta, 1-\delta]
$$

by the trapezoidal rule and imposing periodic BC.

We denote the numerical integration operator with the trapezoidal rule using uniformly distributed nodes as Trp. The function $v_{k}(x)$ stands for the continuous form of the discrete vector $\mathbf{v}_{k}$, which is defined as

$$
v_{k}(x):= \begin{cases}\cos ((x+1)(k-1) \pi), & 1 \leq k \leq \frac{N+1}{2} \\ \sin ((x+1)(k-1) \pi), & \frac{N+1}{2}<k \leq N-1\end{cases}
$$

For rows $\mathbf{b}_{i}, R<i<N-R$ and $k=1, \ldots, N-1$, we have

$$
\begin{aligned}
\mathbf{b}_{i} \cdot \mathbf{v}_{k} & =\operatorname{Trp}\left(\int_{x-\delta}^{x+\delta} v_{k}\left(x^{\prime}\right) d x^{\prime}\right) \\
& =h\left(\frac{1}{2} \mathbf{v}_{k}^{i-R}+\mathbf{v}_{k}^{i-R+1}+\cdots+\mathbf{v}_{k}^{i}+\cdots+\mathbf{v}_{k}^{i+R-1}+\frac{1}{2} \mathbf{v}_{k}^{i+R}\right) .
\end{aligned}
$$

Using simple trigonometric identities such as the sum and difference formulas for $\cos (x)$ and $\sin (x)$, one can show that

$$
\mathbf{b}_{i} \cdot \mathbf{v}_{k}=\lambda_{k}\left(B_{\mathrm{p}}\right) \mathbf{v}_{k}^{i},
$$

where

$$
\lambda_{k}\left(B_{\mathrm{p}}\right)=-h \cos (\delta(k-1) \pi)-h+2 h \sum_{l=0}^{R} \cos (l(k-1) \pi h) .
$$

For rows $\mathbf{b}_{i}, 1 \leq i \leq R$, we have

$$
\mathbf{b}_{i} \cdot \mathbf{v}_{k}=\operatorname{Trp}\left(\int_{-1}^{x+\delta} v_{k}\left(x^{\prime}\right) d x^{\prime}\right)+\operatorname{Trp}\left(\int_{x+2-\delta}^{1} v_{k}\left(x^{\prime}\right) d x^{\prime}\right), \quad x \in[-1,-1+\delta) .
$$


Because $v_{k}(x)=v_{k}(x+2)$, the change of variable $y^{\prime}=x^{\prime}-2$ in the second integral in (15) leads to

$$
\begin{aligned}
\mathbf{b}_{i} \cdot \mathbf{v}_{k} & =\operatorname{Trp}\left(\int_{-1}^{x+\delta} v_{k}\left(x^{\prime}\right) d x^{\prime}\right)+\operatorname{Trp}\left(\int_{x-\delta}^{-1} v_{k}\left(x^{\prime}\right) d x^{\prime}\right) \\
& =\operatorname{Trp}\left(\int_{x-\delta}^{x+\delta} v_{k}\left(x^{\prime}\right) d x^{\prime}\right),
\end{aligned}
$$

which is equivalent to equation (13).

For rows $\mathbf{b}_{i}, N-R<i \leq N-1$, we have

$$
\mathbf{b}_{i} \cdot \mathbf{v}_{k}=\operatorname{Trp}\left(\int_{-1}^{x-2+\delta} v_{k}\left(x^{\prime}\right) d x^{\prime}\right)+\operatorname{Trp}\left(\int_{x-\delta}^{1} v_{k}\left(x^{\prime}\right) d x^{\prime}\right), \quad x \in(1-\delta, 1]
$$

Because $v_{k}(x)=v_{k}(x+2)$, the change of variable $y^{\prime}=x^{\prime}+2$ in the first integral in (17) leads to

$$
\begin{aligned}
\mathbf{b}_{i} \cdot \mathbf{v}_{k} & =\operatorname{Trp}\left(\int_{1}^{x+\delta} v_{k}\left(x^{\prime}\right) d x^{\prime}\right)+\operatorname{Trp}\left(\int_{x-\delta}^{1} v_{k}\left(x^{\prime}\right) d x^{\prime}\right) \\
& =\operatorname{Trp}\left(\int_{x-\delta}^{x+\delta} v_{k}\left(x^{\prime}\right) d x^{\prime}\right),
\end{aligned}
$$

which is equivalent to Equation (13).

We see that the expressions in (12), (16), and (18) are identical. As a result, the eigenvalue expression in (14) holds for all rows $1 \leq i \leq N-1$. Furthermore, it is straightforward to verify (10). Consequently, the vectors $\mathbf{v}_{k}$ in (7) pair with all of the eigenvalues in (8); hence, $\left(\lambda_{k}\left(A_{\mathrm{p}}\right), \mathbf{v}_{k}\right)_{k=1}^{N-1}$ form the eigenpairs of the matrix $A_{\mathrm{p}}$. We used the following trigonometric identities to turn (8) into (9):

$$
h \cos (\delta(k-1) \pi)+h=2 h \frac{(\cos (\delta(k-1) \pi)+1)}{2}=2 h \cos ^{2}\left(\frac{\delta(k-1) \pi}{2}\right),
$$

and

$$
\sum_{l=0}^{R} \cos (l \theta)=\frac{\sin ((R+1) \theta / 2)}{\sin (\theta)} \cos (R \theta / 2),
$$

where $\theta=(k-1) \pi h$ and plugging $R=\frac{\delta}{h}$. Similar identities are used in the proofs of upcoming lemmas.

Now, we find bounds for the spectrum of $A_{\mathrm{p}}$, which will be utilized to determine if Jacobi relaxations converge for the matrix $A_{D}$.

Lemma 2. The eigenvalues of $A_{p}$ satisfy the following bounds:

$$
0 \leq \lambda_{k}\left(A_{\mathrm{p}}\right)<4 \delta-2 h, \quad k=1, \ldots, N-1 .
$$

Proof. It is clear that $\lambda_{1}\left(A_{\mathrm{p}}\right)=0$. Because $\cos (l(k-1) \pi h) \leq 1$ for all $h, l=0, \ldots, R$, and $k=1, \ldots, N-1$, from definition of $\lambda_{k}\left(A_{\mathrm{p}}\right)$ in (8), it follows that $\lambda_{k}\left(A_{\mathrm{p}}\right) \geq 0$. 
For the upper bound, the case of $k=1$ is clear. We proceed with an induction on $R$ where $\delta=R h$, where $R \geq 1$. Then, we need to show that

$$
\lambda_{k}\left(A_{\mathrm{p}}\right)=2 \delta-h \sin ((k-1) \pi \delta) \cot ((k-1) \pi h / 2)<4 \delta-2 h, \quad k=2, \ldots, N-1 .
$$

The above inequality is equivalent to showing that

$$
\sin ((k-1) \pi R h) \cot ((k-1) \pi h / 2)>2-2 R .
$$

We immediately see that the case of $R=1$ holds, that is,

$$
\sin ((k-1) \pi h) \cot ((k-1) \pi h / 2)>0 .
$$

Now, assume that (19) holds for $R=m>1$. Namely,

$$
\sin ((k-1) \pi m h) \cot ((k-1) \pi h / 2)>2-2 m, \quad k=2, \ldots, N-1 .
$$

We will show that (19) holds for $R=m+1$. Using (20), (21), and the fact that $\cos (x) \leq 1$, we arrive at

$$
\begin{aligned}
\sin ((k-1) \pi(m+1) h) \cot ((k-1) \pi h / 2)= & \sin ((k-1) \pi h m) \cos ((k-1) \pi h) \cot ((k-1) \pi h / 2) \\
& +\sin ((k-1) \pi h) \cos ((k-1) \pi h m) \cot ((k-1) \pi h / 2) \\
\geq & \sin ((k-1) \pi h m) \cot ((k-1) \pi h / 2)+\sin ((k-1) \pi h) \cot ((k-1) \pi h / 2) \\
> & 2-2 m+0 \\
> & 2-2(m+1) .
\end{aligned}
$$

This completes the induction step.

\section{2 | Antiperiodic matrix $A_{a}$}

The matrix $A_{\mathrm{a}}$ is symmetric positive definite and Toeplitz. We present its eigenpairs.

Lemma 3. The matrix $A_{a}$ assumes the eigenvectors, for $1 \leq i \leq N-1$,

$$
\mathbf{v}_{k}^{i}:= \begin{cases}\cos \left(\frac{(i-1)\left(k-\frac{1}{2}\right) 2 \pi}{N-1}\right), & 1 \leq k \leq \frac{N+1}{2}, \\ \sin \left(\frac{(i-1)\left(k-\frac{1}{2}\right) 2 \pi}{N-1}\right), & \frac{N+1}{2}<k \leq N-1,\end{cases}
$$

with the corresponding eigenvalues where $h:=\frac{2}{N-1}$

$$
\begin{aligned}
\lambda_{k}\left(A_{\mathrm{a}}\right) & :=2 \delta+h \cos \left(\delta\left(k-\frac{1}{2}\right) \pi\right)+h-2 h \sum_{l=0}^{R} \cos \left(l\left(k-\frac{1}{2}\right) \pi h\right) \\
& =2 \delta-h \frac{\sin \left(\left(k-\frac{1}{2}\right) \pi \delta\right) \sin \left(\left(k-\frac{1}{2}\right) \pi h\right)}{2 \sin ^{2}\left(\frac{\left(k-\frac{1}{2}\right) \pi h}{2}\right)}, \quad k=1, \ldots, N-1 .
\end{aligned}
$$


The eigenvalues have the property, for $k=1, \ldots,(N-1) / 2$,

$$
\lambda_{k}\left(A_{\mathrm{a}}\right)=\lambda_{N-k}\left(A_{\mathrm{a}}\right) .
$$

Hence, the eigenvalues have geometric multiplicity 2.

Proof. Note that the vectors in (22) are orthogonal:

$$
\mathbf{v}_{k} \cdot \mathbf{v}_{l}:= \begin{cases}0, & 1 \leq k \neq l \leq N-1, \\ \frac{N-1}{2}, & 1 \leq k=l \leq N-1 .\end{cases}
$$

As in the proof of Lemma 1 for the periodic case, we define the shifted matrix $B_{a}:=2 \delta I-A_{a}$. Depending on which interval $x$ comes from in (5), the rows $\mathbf{b}_{i}$ of $B_{a}$ originate from three different integral equations. We apply the same procedure in Lemma 1 and conclude that the vectors $\mathbf{v}_{k}$ in (22) pair with all of the eigenvalues in (23); hence, $\left(\lambda_{k}\left(A_{\mathrm{a}}\right), \mathbf{v}_{k}\right)_{k=1}^{N-1}$ form the eigenpairs of the matrix $A_{\mathrm{a}}$.

Now, we find bounds for the spectrum of $A_{a}$, which will be utilized to determine if Jacobi relaxations converge.

Lemma 4. The eigenvalues of $A_{a}$ satisfy the following bounds:

$$
0<\lambda_{k}\left(A_{\mathrm{a}}\right)<4 \delta-2 h, \quad k=1, \ldots, N-1 .
$$

Proof. Because $0<\left(k-\frac{1}{2}\right) \pi h<2 \pi$ for $k=1,2, \ldots, N-1$, the terms in $(23) \cos \left(l\left(k-\frac{1}{2}\right) \pi h\right)$ cannot be equal to 1 for a fixed $k$, simultaneously. Therefore,

$$
\lambda_{k}\left(A_{\mathrm{a}}\right)>2 \delta+h+h-2 h\left(\frac{\delta}{h}+1\right)=0 .
$$

Applying the same steps in Lemma 2, we can show that

$$
\lambda_{k}\left(A_{\mathrm{a}}\right)<4 \delta-2 h .
$$

\section{3 | Dirichlet matrix $A_{\mathrm{D}}$}

The matrix $A_{\mathrm{D}}$ is symmetric positive definite. Unlike the matrices $A_{\mathrm{p}}$ and $A_{\mathrm{a}}$, it is not Toeplitz. We present its eigenpairs.

Lemma 5. The matrix $A_{D}$ assumes the eigenvectors, for $1 \leq i \leq N-2$,

$$
\mathbf{v}_{k}^{i}:= \begin{cases}\sin \left(\frac{i k 2 \pi}{N-1}\right), & 1 \leq k<\frac{N-1}{2}, \\ \sin \left(\frac{i\left(k+\frac{1}{2}\right) 2 \pi}{N-1}\right), & \frac{N-1}{2} \leq k \leq N-2,\end{cases}
$$

with the corresponding eigenvalues where $h:=\frac{2}{N-1}$,

$$
\begin{aligned}
\lambda_{k}\left(A_{\mathrm{D}}\right): & = \begin{cases}2 \delta+h \cos (\delta k \pi)+h-2 h \sum_{l=0}^{R} \cos (l k \pi h), & 1 \leq k<\frac{N-1}{2} \\
2 \delta+h \cos \left(\delta\left(k+\frac{1}{2}\right) \pi\right)+h-2 h \sum_{l=0}^{R} \cos \left(l\left(k+\frac{1}{2}\right) \pi h\right), & \frac{N-1}{2} \leq k \leq N-2 .\end{cases} \\
& = \begin{cases}2 \delta-h \frac{\sin (k \pi \delta) \sin (k \pi h)}{2 \sin ^{2}\left(\frac{k \pi h}{2}\right)}, & 1 \leq k<\frac{N-1}{2} \\
2 \delta-h \frac{\sin \left(\left(k+\frac{1}{2}\right) \pi \delta\right) \sin \left(\left(k+\frac{1}{2}\right) \pi h\right)}{2 \sin ^{2}\left(\frac{\left(k+\frac{1}{2}\right) \pi h}{2}\right)}, & \frac{N-1}{2} \leq k \leq N-2 .\end{cases}
\end{aligned}
$$


Proof. Note that the vectors in (5) are orthogonal:

$$
\mathbf{v}_{k} \cdot \mathbf{v}_{l}:= \begin{cases}0, & 1 \leq k \neq l \leq N-2 \\ \frac{N-1}{2}, & 1 \leq k=l \leq N-2\end{cases}
$$

As in the proof of Lemma 1 for the periodic case, we define the shifted matrix $B_{\mathrm{D}}:=2 \delta I-A_{\mathrm{D}}$. Depending on which interval $x$ comes from in (6), the rows $\mathbf{b}_{i}$ of $B_{\mathrm{D}}$ originate from three different integral equations. The rows $\mathbf{b}_{i}, R \leq i \leq$ $N-R-1$ are less involved because they are obtained from the discretization of the operator

$$
\int_{x-\delta}^{x+\delta} u\left(x^{\prime}\right) d x^{\prime}=f, \quad x \in[-1+\delta, 1-\delta]
$$

by the trapezoidal rule and imposing Dirichlet BC.

The continuous form $v_{k}(x)$ of the discrete vector $\mathbf{v}_{k}$ is defined as

$$
v_{k}(x):= \begin{cases}\sin ((x+1) k \pi), & 1 \leq k<\frac{N-1}{2} \\ \sin \left((x+1)\left(k+\frac{1}{2}\right) \pi\right), & \frac{N-1}{2} \leq k \leq N-2\end{cases}
$$

First, we consider the case for $1 \leq k<\frac{N-1}{2}$ for which $v_{k}(x)$ is odd. Because $v_{k}(-1)=v_{k}(1)=0$, we have

$$
\mathbf{b}_{i+1} \cdot \mathbf{v}_{k}=\operatorname{Trp}\left(\int_{x-\delta}^{x+\delta} v_{k}\left(x^{\prime}\right) d x^{\prime}\right), \quad k=1, \ldots, \frac{N-1}{2} .
$$

The eigenfunctions $v_{k}(x)$ coincide with those with periodic BC. Using (14), we obtain $\mathbf{b}_{i} \cdot \mathbf{v}_{k}=\left(2 \delta-\lambda_{k}\right) \mathbf{v}_{k}^{i}$ where $\lambda_{k}$ is defined in (24).

For rows $\mathbf{b}_{i}, 1 \leq i<R$, because $v_{k}(-1)=v_{k}(1)=0$, we have

$$
\mathbf{b}_{i} \cdot \mathbf{v}_{k}=\operatorname{Trp}\left(\int_{-1}^{x+\delta} v_{k}\left(x^{\prime}\right) d x^{\prime}\right)-\operatorname{Trp}\left(\int_{-1}^{-x+\delta-2} v_{k}\left(x^{\prime}\right) d x^{\prime}\right), \quad x \in[-1,-1+\delta) .
$$

Using the oddness of the eigenfunctions, that is, $v_{k}(-x)=-v_{k}(x)$, and the change of variable $x^{\prime}=-y^{\prime}-2$ in the second integral in (26) leads to

$$
\begin{aligned}
\mathbf{b}_{i} \cdot \mathbf{v}_{k} & =\operatorname{Trp}\left(\int_{-1}^{x+\delta} v_{k}\left(x^{\prime}\right) d x^{\prime}\right)+\operatorname{Trp}\left(\int_{x-\delta}^{-1} v_{k}\left(x^{\prime}\right) d x^{\prime}\right) \\
& =\operatorname{Trp}\left(\int_{x-\delta}^{x+\delta} v_{k}\left(x^{\prime}\right) d x^{\prime}\right),
\end{aligned}
$$

which is equivalent to Equation (25).

For rows $\mathbf{b}_{i}, N-R-1<i \leq N-2$, we have

$$
\mathbf{b}_{i} \cdot \mathbf{v}_{k}=-\operatorname{Trp}\left(\int_{-x-\delta+2}^{1} v_{k}\left(x^{\prime}\right) d x^{\prime}\right)+\operatorname{Trp}\left(\int_{x-\delta}^{1} v_{k}\left(x^{\prime}\right) d x^{\prime}\right), \quad x \in(1-\delta, 1] .
$$


Using the oddness of the eigenfunctions, $v_{k}(-1)=v_{k}(1)=0$, and the change of variable $x^{\prime}=2-y^{\prime}$ in the first integral in (28) leads to

$$
\begin{aligned}
\mathbf{b}_{i} \cdot \mathbf{v}_{k} & =\operatorname{Trp}\left(\int_{1}^{x+\delta} v_{k}\left(x^{\prime}\right) d x^{\prime}\right)+\operatorname{Trp}\left(\int_{x-\delta}^{1} v_{k}\left(x^{\prime}\right) d x^{\prime}\right) \\
& =\operatorname{Trp}\left(\int_{x-\delta}^{x+\delta} v_{k}\left(x^{\prime}\right) d x^{\prime}\right),
\end{aligned}
$$

which is equivalent to Equation (25).

We see that the expressions in (25), (27), and (29) are identical. The case for $\frac{N-1}{2} \leq k \leq N-2$ for which $v_{k}(x)$ is even give rise to identical results. Consequently, the vectors $\mathbf{v}_{k}$ in (5) pair with all of the eigenvalues in (24); hence, $\left(\lambda_{k}\left(A_{\triangleright}\right), \mathbf{v}_{k}\right)_{k=1}^{N-2}$ form the eigenpairs of the matrix $A_{\triangleright}$.

\section{4 | CONSTRUCTION OF SMOOTHERS FOR NONLOCAL PROBLEMS}

We consider the linear system of equation

$$
A_{\mathrm{BC}} \mathbf{u}=\mathbf{f},
$$

where $\mathrm{BC}=\{\mathrm{a}, \mathrm{D}\}$. These matrices are invertible. Let $\mathbf{u}$ and $\mathbf{v}$ denote the exact and an approximate solution to (30), respectively. In addition, let $\mathbf{e}$ and $\mathbf{r}$ denote the error and the residual, respectively, defined by

$$
\begin{aligned}
& \mathbf{e}:=\mathbf{u}-\mathbf{v}, \\
& \mathbf{r}:=\mathbf{f}-A_{\mathrm{BC}} \mathbf{v} .
\end{aligned}
$$

We examine stationary relaxation schemes, in particular Jacobi relaxation, applied to systems (30). In general, these schemes are not preferred as solvers to Fredholm second kind systems due to slow convergence because the system matrices are usually far from being diagonally dominant.

We begin with studying Jacobi relaxation for the matrix $A_{\mathrm{a}}$. We note that $A_{\mathrm{a}}$ is constant along the diagonal. This property allows us to obtain eigenvalues of the iteration matrix explicitly. We express the Jacobi relaxation in matrix form by using the following splitting:

$$
A_{\mathrm{a}}=D_{\mathrm{a}}-L_{\mathrm{a}}-U_{\mathrm{a}},
$$

where $D_{\mathrm{a}},-L_{\mathrm{a}}$, and $-U_{\mathrm{a}}$ denote the diagonal, strictly lower triangular, and upper triangular parts of $A_{\mathrm{a}}$, respectively.

We arrange $A_{\mathrm{a}} \mathbf{u}=\mathbf{f}$ as

$$
\mathbf{u}=D_{\mathrm{a}}^{-1}\left(D_{\mathrm{a}}-A_{\mathrm{a}}\right) \mathbf{u}+D_{\mathrm{a}}^{-1} \mathbf{f}=\left(I-D_{\mathrm{a}}^{-1} A_{\mathrm{a}}\right) \mathbf{u}+D_{\mathrm{a}}^{-1} \mathbf{f},
$$

and define the Jacobi iteration matrix by

$$
G_{\mathrm{a}}^{J}:=I-D_{\mathrm{a}}^{-1} A_{\mathrm{a}} .
$$

Then, we define the Jacobi relaxation in matrix form as

$$
\mathbf{v}^{1}=G_{\mathrm{a}}^{J} \mathbf{v}^{0}+D_{\mathrm{a}}^{-1} \mathbf{f}
$$


A slight modification of Jacobi relaxation leads to the weighted (damped) Jacobi relaxation as

$$
\mathbf{v}^{1}=\left[(1-\omega) I+\omega G_{\mathrm{a}}^{J}\right] \mathbf{v}^{0}+\omega D_{\mathrm{a}}^{-1} \mathbf{f}
$$

When we define the weighted Jacobi iteration matrix as

$$
G_{a}^{\omega}:=(1-\omega) I+\omega G_{a}^{J},
$$

then the method becomes

$$
\mathbf{v}^{1}=G_{a}^{\omega} \mathbf{v}^{0}+\omega D_{a}^{-1} \mathbf{f} .
$$

Because the matrix $A_{\mathrm{a}}$ is constant along the diagonal with entries $2 \delta-h$, we can easily obtain the eigenvalues of the iteration matrices $G_{\mathrm{a}}^{J}$ and $G_{\mathrm{a}}^{\omega}$ :

$$
\begin{aligned}
\lambda_{k}\left(G_{a}^{J}\right) & =1-\frac{1}{2 \delta-h} \lambda_{k}\left(A_{a}\right), \\
\lambda_{k}\left(G_{a}^{\omega}\right) & =(1-\omega)+\omega \lambda_{k}\left(G_{a}^{J}\right) .
\end{aligned}
$$

It follows from the Lemma 4 that $\rho\left(G_{a}^{J}\right)<1$ and $\rho\left(G_{a}^{\omega}\right)<1$. Hence, Jacobi and weighted Jacobi relaxations converge for $0<\omega \leq 1$.

Now, we discuss the action of the iteration matrix on $G_{a}^{\omega}$ on the smooth modes. Note that Fourier modes (eigenvectors of $A_{a}$ ) in the first quarter and last quarter of the spectrum, that is, modes with wavenumbers in the ranges $1 \leq k \leq \frac{N-1}{4}$ and $\frac{3(N-1)}{4}<k \leq N-1$ are classified as low-frequency or smooth modes. However, the modes at the center of the spectrum with wavenumbers in the range $\frac{N-1}{4}<k \leq \frac{3(N-1)}{4}$ are classified as high-frequency or oscillatory modes.

Let $\mathbf{e}^{0}$ be the initial error and it can be represented in terms of eigenvectors of $A_{\mathrm{a}}$ as

$$
\mathbf{e}^{0}=\sum_{k=1}^{N-1} c_{k} \mathbf{v}_{\mathbf{k}}
$$

After $m$ iterations with $G_{a}^{\omega}$, the error becomes

$$
\mathbf{e}^{m}=G_{a}^{\omega} \mathbf{e}^{0}=\sum_{k=1}^{N-1} c_{k}\left(G_{a}^{\omega}\right)^{m} \mathbf{v}_{\mathbf{k}}=\sum_{k=1}^{N-1} c_{k} \lambda_{k}^{m}\left(G_{a}^{\omega}\right) \mathbf{v}_{\mathbf{k}},
$$

thereby reducing the initial error of the $k$ th mode by a factor of $\lambda_{k}^{m}\left(G_{a}^{\omega}\right)$.

We start analyzing weighted Jacobi relaxation by quantifying the eigenvalues using a Taylor expansion corresponding to smoothest modes:

$$
\lambda_{1}\left(A_{\mathrm{a}}\right)=\lambda_{N-1}\left(A_{\mathrm{a}}\right)=\frac{\pi^{2} \delta^{3}}{6}+\frac{\pi^{2} \delta h^{2}}{12}+\mathcal{O}\left(h \delta^{4}\right)+\mathcal{O}\left(h^{4}\right) .
$$

Because $\delta>h$ and the term $\frac{\pi^{2} \delta^{3}}{6}$ dominates, we have

$$
\lambda_{1}\left(A_{\mathrm{a}}\right)=\lambda_{N-1}\left(A_{\mathrm{a}}\right)=\mathcal{O}\left(\delta^{3}\right) .
$$

It follows from these observations that for $\omega$ satisfying $0<\omega \leq 1$, we obtain

$$
\lambda_{1}\left(G_{a}^{\omega}\right)=\lambda_{N-1}\left(G_{a}^{\omega}\right)=1+\mathcal{O}\left(\delta^{2}\right) .
$$



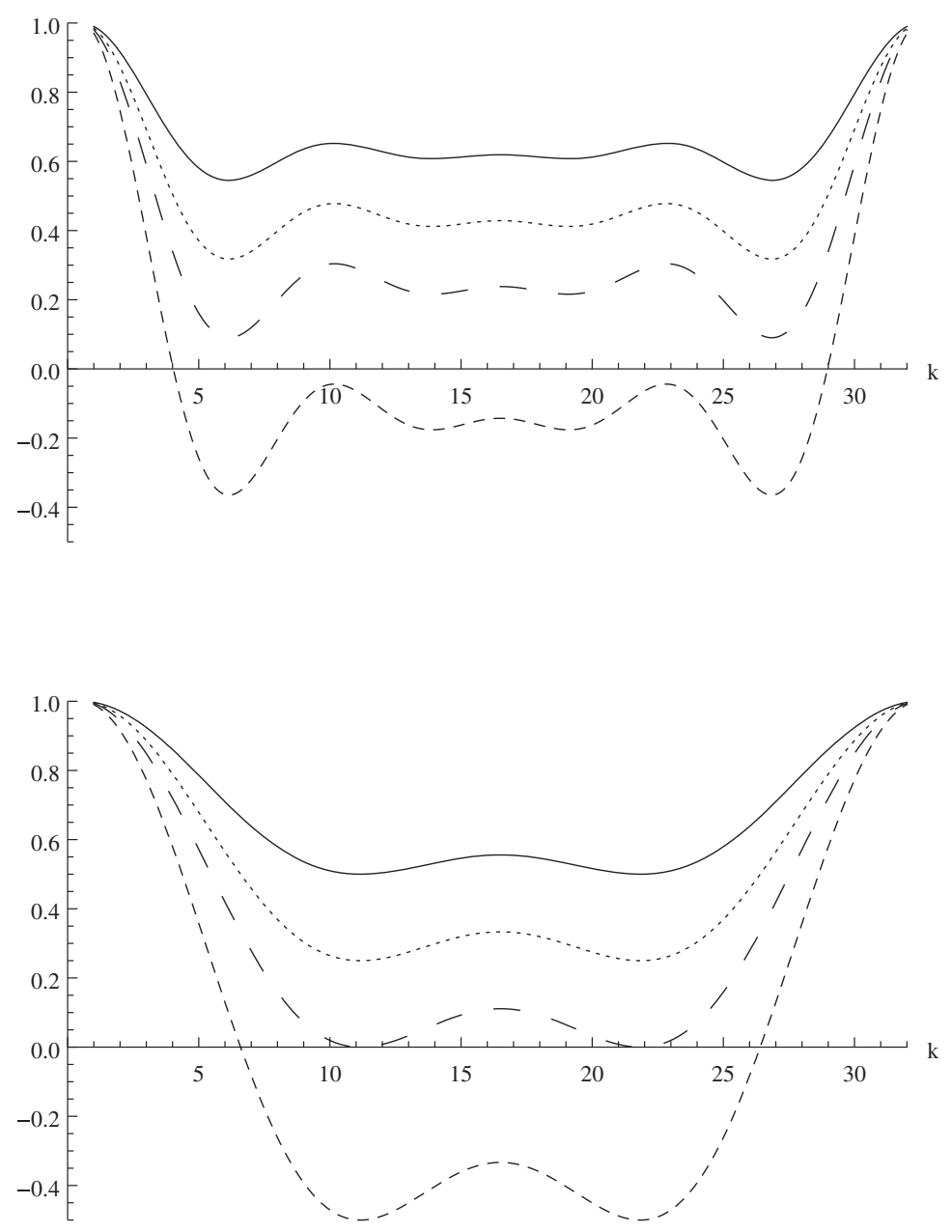

$$
\begin{aligned}
& \cdots \quad \omega=1 \\
& -\quad \omega=2 / 3 \\
& \cdots \cdots=1 / 2 \\
& \ldots \omega=1 / 3
\end{aligned}
$$$$
--\omega=1
$$$$
-\omega=2 / 3
$$$$
\cdots \omega=1 / 2
$$$$
\omega=1 / 3
$$

FIGURE 1 Eigenvalues of the iteration matrix $G_{\mathrm{a}}^{\omega}$ for $\omega=1,2 / 3,1 / 2,1 / 3$ for $N=33$ and $\delta=0.25$

FIGURE 2 Eigenvalues of the iteration matrix $G_{a}^{\omega}$ for $\omega=1,2 / 3,1 / 2,1 / 3$ for $N=33$ and $\delta=0.125$

The smaller $\delta$ is, the closer $\lambda_{1}\left(G_{a}^{\omega}\right)$ and $\lambda_{N-1}\left(G_{a}^{\omega}\right)$ are to 1 . Hence, $\lambda_{1}\left(G_{a}^{\omega}\right)$ and $\lambda_{N-1}\left(G_{a}^{\omega}\right)$ are close to 1 . Finally, we conclude that no value of $\omega$ will damp the smooth components of the error quickly. This is the expected behavior because smoothers are designed to damp the oscillatory modes, not the smooth ones. We depict this fact in Figures 1, 2 for the values of $\delta=0.25$ and $\delta=0.125$, respectively.

Having observed that no value of $\omega$ reduces the smooth components effectively, we try to find the best choices of $\omega$ that damp the oscillatory modes effectively. To accomplish this, we propose two strategies.

\section{1 $\mid$ The first strategy}

We focus only on the most oscillatory mode. We aim to damp it as quickly as possible by minimizing the absolute value of eigenvalue of $G_{\mathrm{a}}^{\omega}$ corresponding to most oscillatory mode. By assuming that $\lambda_{k}\left(G_{\mathrm{a}}^{\omega}\right)$ is continuous in $k$, we want to enforce

$$
\lambda_{\frac{N}{2}}\left(G_{a}^{\omega}\right)=0,
$$

and present the appropriate choice of $\omega$ for doing that.

Lemma 6. Assume that $\lambda_{k}\left(G_{\mathrm{a}}^{\omega}\right)$ is continuous in $k$. Then, $\lambda_{\frac{N}{2}}\left(G_{\mathrm{a}}^{\omega}\right)=0$ implies

$$
\omega=1-\frac{h}{2 \delta}
$$


Proof. We have two cases. First, we assume that $R$ is an odd integer. Then,

$$
\begin{aligned}
\lambda_{\frac{N}{2}}\left(G_{a}^{\omega}\right) & =1-\omega+\omega\left(1-\frac{2 \delta+h \cos \left(\frac{\delta \pi}{h}\right)+h-2 h \sum_{l=0}^{R} \cos (l \pi)}{2 \delta-h}\right) \\
& =1-\omega+\omega\left(\frac{2 \delta-h-2 \delta-h \cos \left(\frac{\delta \pi}{h}\right)-h}{2 \delta-h}\right) \\
& =0 .
\end{aligned}
$$

It follows that

$$
\omega=\frac{1}{1+\frac{2 h+h \cos \left(\frac{\delta \pi}{h}\right)}{2 \delta-h}}=1-\frac{h}{2 \delta} .
$$

Now, assume that $R$ is an even integer. Then,

$$
\lambda_{\frac{N}{2}}\left(G_{a}^{\omega}\right)=1-\omega+\omega\left(\frac{-h \cos \left(\frac{\delta \pi}{h}\right)}{2 \delta-h}\right)=0 .
$$

This implies that

$$
\omega=\frac{1}{1+\frac{h \cos \left(\frac{\delta \pi}{h}\right)}{2 \delta-h}}=1-\frac{h}{2 \delta} .
$$

In Figure 3, we depict the eigenvalues of $G_{a}^{\omega}$ for $\delta=0.5,0.25,0.125$ when $\omega=1-\frac{h}{2 \delta}$ and $N=33$. We observe that the eigenvalue associated to the most oscillatory mode is very close to 0 . In addition, the eigenvalues associated to oscillatory modes (those with $\frac{N-1}{4}<k \leq \frac{3(N-1)}{4}$ ) are also close to 0 . As a result, for $\omega=1-\frac{h}{2 \delta}$, although smooth component of the error reduce slowly, oscillatory components are damped rapidly for any $\delta$ satisfying $0<\delta<1$.

We want to investigate if the first strategy is related to the Picard iteration in any way. Let us recall the weighted Jacobi relaxation

$$
\mathbf{v}^{1}=G_{\mathrm{a}}^{\omega} \mathbf{v}^{0}+\omega D_{\mathrm{a}}^{-1} \mathbf{f}=\left((1-\omega) I+\omega\left(I-D_{\mathrm{a}}^{-1} A_{\mathrm{a}}\right)\right) \mathbf{v}^{0}+\omega D_{\mathrm{a}}^{-1} \mathbf{f} .
$$

Substituting $\omega=1-\frac{h}{2 \delta}$ in (32), the weighted Jacobi relaxation turns into

$$
\begin{aligned}
\mathbf{v}^{1} & =\left(\left(1-1+\frac{h}{2 \delta}\right) I+\left(1-\frac{h}{2 \delta}\right)\left(I-D_{\mathrm{a}}^{-1} A_{\mathrm{a}}\right)\right) \mathbf{v}^{0}+\left(1-\frac{h}{2 \delta}\right) D_{\mathrm{a}}^{-1} \mathbf{f} \\
& =\left(I-\frac{1}{2 \delta} A_{\mathrm{a}}\right) \mathbf{v}^{0}+\frac{1}{2 \delta} \mathbf{f} .
\end{aligned}
$$

This iteration is nothing but the Picard iteration. In other words, for $\omega=1-\frac{h}{2 \delta}$ weighted Jacobi is equivalent to the Picard iteration. Consequently, our investigation revealed the working principle of the Picard iteration from a spectral point of view.

Let us see if the same working principle of the Picard iteration is also valid for the Dirichlet matrix $A_{\mathrm{D}}$. First, note that the matrix $A_{\mathrm{D}}$ is not constant along the diagonal. Therefore, it is difficult to directly apply the same spectral analysis done for the Jacobi relaxation using the matrix $A_{a}$. However, if we switch to Jacobi-like relaxation by setting the diagonal matrix to $D_{\mathrm{D}}=(2 \delta-h) I$ in the splitting $A_{\mathrm{D}}=D_{\mathrm{D}}+L_{\mathrm{D}}+U_{\mathrm{D}}$, we can easily transfer the analysis done for $A_{\mathrm{a}}$. However, we skip this approach and instead, we examine the Picard iteration applied to $A_{\mathrm{D}}$. This is a more direct way to find out if the Picard iteration is consistent with the first strategy. 


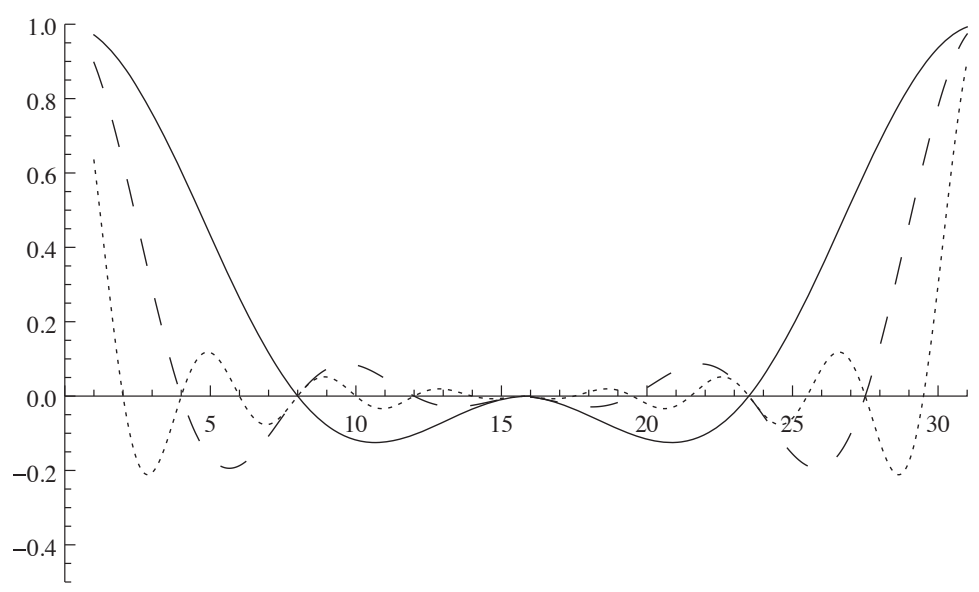

FIGURE 3 Eigenvalues of the iteration matrix $G_{a}^{\omega}$ for $\delta=0.5,0.25,0.125$ when $\omega=1-\frac{h}{2 \delta}$ and $N=33$

Let us recall the Picard iteration applied to $A_{\mathrm{D}}$ :

$$
\mathbf{v}^{1}=\left(I-\frac{1}{2 \delta} A_{\mathrm{D}}\right) \mathbf{v}^{0}+\frac{1}{2 \delta} \mathbf{f} .
$$

Similar to $A_{\mathrm{a}}$, the eigenvector of $A_{\mathrm{D}}$ in the range $1 \leq k \leq \frac{N-1}{4}$ and $\frac{3(N-1)}{4} \leq k \leq N-2$ are smooth modes, whereas the ones in the range $\frac{N-1}{4}<k<\frac{3(N-1)}{4}$ are oscillatory. The eigenvalues of the iteration matrix of the Picard iteration $G_{\mathrm{D}}^{P}$ are

$$
\lambda_{k}\left(G_{\mathrm{D}}^{p}\right)=1-\frac{1}{2 \delta} \lambda_{k}\left(A_{\mathrm{D}}\right), \quad k=1, \ldots, N-2 .
$$

The matrix $A_{\mathrm{D}}$ assumes all eigenvalues of the matrix $A_{\mathrm{a}}$ and $A_{\mathrm{p}}$ except $\lambda_{1}\left(A_{\mathrm{p}}\right)=0$. From Lemmas 2 and 4 , it follows that

$$
\rho\left(G_{\mathrm{D}}^{P}\right)<1
$$

which indicates that the Picard iteration converges.

Notice that for smoothest modes

$$
\begin{aligned}
\lambda_{1}\left(A_{\mathrm{D}}\right) & =\mathcal{O}\left(\delta^{3}\right) \\
\lambda_{N-2}\left(A_{\mathrm{D}}\right) & =\lambda_{N-1}\left(A_{а}\right)=\mathcal{O}\left(\delta^{3}\right) .
\end{aligned}
$$

Hence, it follows that

$$
\begin{aligned}
\lambda_{1}\left(G_{\mathrm{D}}^{P}\right) & =1+\mathcal{O}\left(\delta^{2}\right) \\
\lambda_{N-2}\left(G_{\mathrm{D}}^{P}\right) & =1+\mathcal{O}\left(\delta^{2}\right) .
\end{aligned}
$$

For most oscillatory modes, because eigenvalues of $A_{\mathrm{D}}$ agree with those of $A_{\mathrm{p}}$, we resort to the eigenvalues of $A_{\mathrm{p}}$. It is easy to show that $\lambda_{\frac{N-1}{2}}\left(A_{\mathrm{p}}\right)=2 \delta$. From the definitions of $A_{\mathrm{D}}$, we can infer that $\lambda_{\frac{N-3}{2}}\left(A_{\mathrm{D}}\right)$ and $\lambda_{\frac{N-1}{2}}\left(A_{\mathrm{D}}\right)$ are close to $2 \delta$. Hence, $\lambda_{\frac{N-3}{2}}\left(G_{\mathrm{D}}^{P}\right)$ and $\lambda_{\frac{N-1}{2}}\left(G_{\mathrm{D}}^{P}\right)$ are close to 0 . In summary, we showed that the two most oscillatory modes can be damped rapidly, whereas the two smoothest modes cannot. In Figure 4, we depict the eigenvalues of $G_{\mathrm{D}}^{P}$ for $\delta=0.5,0.25,0.125$ when $N=33$. Consequently, our investigation reveals that the working principle of the Picard iteration for $A_{\mathrm{a}}$ is also valid for the matrix $A_{\mathrm{D}}$. 
FIGURE 4 Eigenvalues of the iteration matrix $G_{\mathrm{D}}^{P}$ for $\delta=0.5,0.25,0.125$ when $N=33$

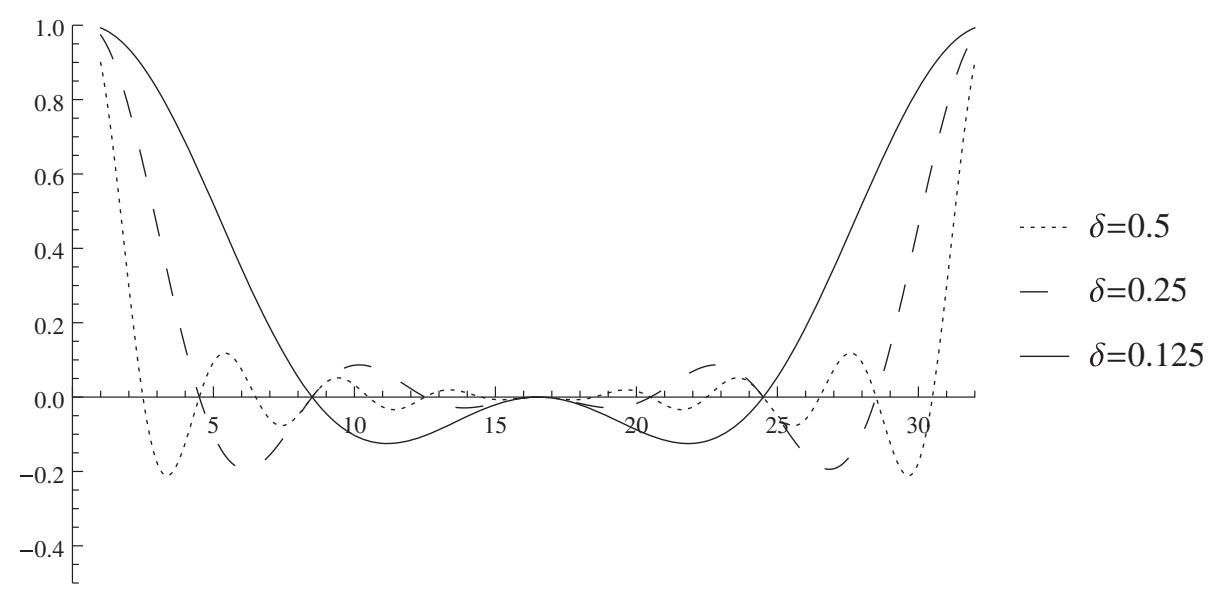

\subsection{The second strategy}

In this strategy, we focus on the oscillatory modes of the weighted Jacobi relaxation. Our aim is to damp them simultaneously as rapidly as possible. Eigenvalues corresponding to oscillatory modes reside at the center of the spectrum. Actually, the same strategy is applied to 1D Poisson problem..$^{20,21}$ For this strategy, it is not straightforward to find a parameter for weighted Jacobi explicitly, but we provide a way to numerically find an optimal parameter with given $\delta$ and $h$.

Assume that $\lambda_{k}\left(A_{\mathrm{a}}\right), \lambda_{k}\left(A_{\mathrm{D}}\right), \lambda_{k}\left(G_{\mathrm{a}}^{\omega}\right)$, and $\lambda_{k}\left(G_{\mathrm{D}}^{\omega}\right)$ are continuous in $k$. The eigenvalues of $\lambda_{k}\left(G_{\mathrm{a}}^{\omega}\right)$ are obtained by adding a constant and multiplying $\lambda_{k}\left(A_{\mathrm{a}}\right)$ with another constant; see (31). Therefore, the critical points of $\lambda_{k}\left(G_{a}^{\omega}\right)$ and $\lambda_{k}\left(G_{\mathrm{D}}^{\omega}\right)$ do not change with respect to $\omega$ and are the same as those of $\lambda_{k}\left(A_{a}\right)$ and $\lambda_{k}\left(A_{\mathrm{D}}\right)$, respectively; see Figures 1 and 2. Because these ideas easily carry to the case of $A_{\mathrm{D}}$, we proceed with the case of $A_{\mathrm{a}}$. Recall that modes with wavenumbers in the range $\frac{N-1}{4}<k \leq \frac{3(N-1)}{4}$ correspond to oscillatory modes. Let

$$
\bar{\lambda}_{k}\left(A_{\mathrm{a}}\right):=\max _{\frac{N-1}{4}<k \leq \frac{3(N-1)}{4}} \lambda_{k}\left(A_{\mathrm{a}}\right) \quad \text { and } \quad \underline{\lambda}_{k}\left(A_{\mathrm{a}}\right):=\min _{\frac{N-1}{4}<k \leq \frac{3(N-1)}{4}} \lambda_{k}\left(A_{\mathrm{a}}\right) .
$$

Because we aim to damp a group of modes, we accomplish this by setting

$$
\bar{\lambda}_{k}\left(G_{a}^{\omega}\right)=-\underline{\lambda}_{k}\left(G_{a}^{\omega}\right)
$$

in order to find optimal value of $\omega$. More precisely,

$$
1-\frac{\omega}{2 \delta-h} \bar{\lambda}_{k}\left(A_{\mathrm{a}}\right)=-1+\frac{\omega}{2 \delta-h} \underline{\lambda}_{k}\left(A_{\mathrm{a}}\right) .
$$

Solving above equation for $\omega$, we obtain the optimal value of $\omega$ as

$$
\omega=\frac{2(2 \delta-h)}{\bar{\lambda}_{k}\left(A_{\mathrm{a}}\right)+\underline{\lambda}_{k}\left(A_{\mathrm{a}}\right)} .
$$

Again, by choosing $D_{\mathrm{D}}=(2 \delta-h) I$ in the splitting

$$
A_{\mathrm{D}}=D_{\mathrm{D}}+L_{\mathrm{D}}+U_{\mathrm{D}}
$$

the optimal value of $\omega$ can be found similarly for $A_{D}$ as

$$
\omega=\frac{2(2 \delta-h)}{\bar{\lambda}_{k}\left(A_{\mathrm{D}}\right)+\underline{\lambda}_{k}\left(A_{\mathrm{D}}\right)} .
$$




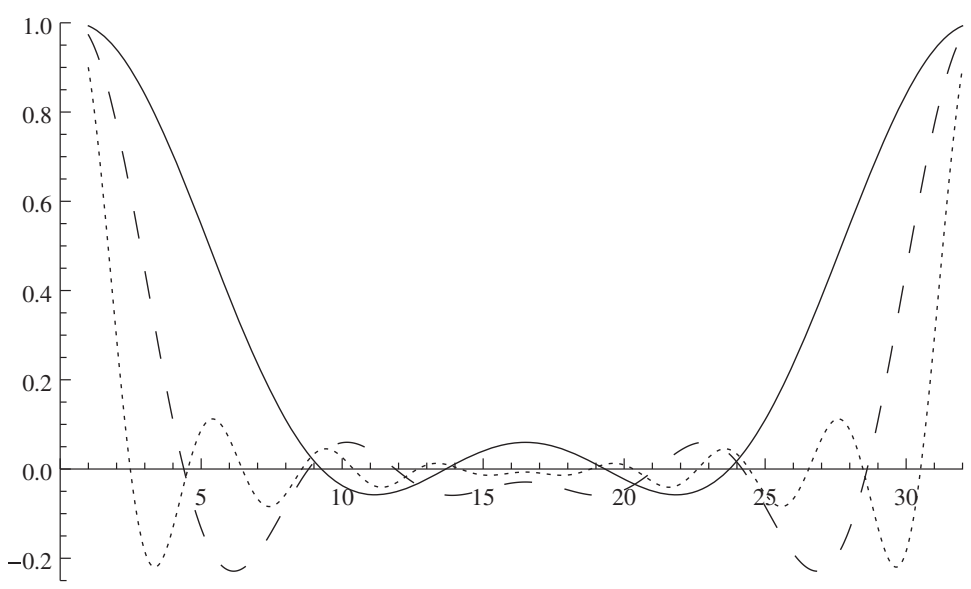

$\cdots \quad \delta=0.50 \Longrightarrow \omega=0.9439$

$-\quad \delta=0.25 \Longrightarrow \omega=0.9005$

$-\delta=0.125 \Longrightarrow \omega=0.7052$

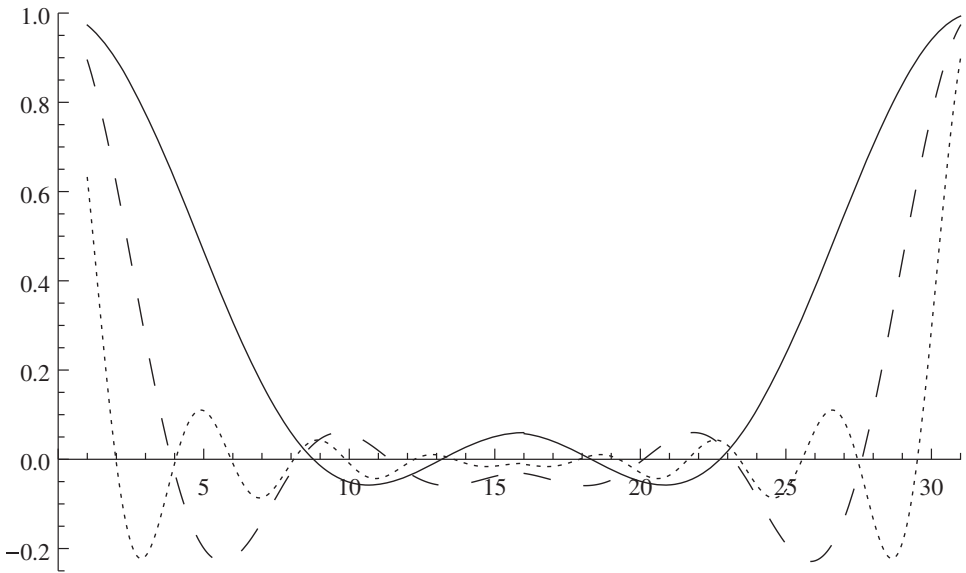

$\cdots . . \quad \delta=0.50 \Longrightarrow \omega=0.9460$

$-\quad \delta=0.25 \Longrightarrow \omega=0.9005$

$-\delta=0.125 \Longrightarrow \omega=0.7052$

FIGURE 5 Eigenvalues of the iteration matrix $G_{\mathrm{a}}^{\omega}(\mathrm{up})$ and $G_{\mathrm{D}}^{\omega}($ down) for $\delta=0.5,0.25,0.125$ when $N=33$

In Figure 5, we show the eigenvalues of $G_{a}^{\omega}$ and $G_{\mathrm{D}}^{\omega}$ for different values of $\delta(\delta=0.5,0.25,0.125)$ when $N=33$ and give the corresponding values of $\omega$. Graphs indicate that our proposed way of finding the optimal $\omega$ works successfully.

\section{5 | NUMERICAL COMPARISON OF THE TWO STRATEGIES}

We make comparisons between the two strategies. We consider only the matrix $A_{\mathrm{D}}$ because results for $A_{\mathrm{a}}$ are similar. The smoother is critical in obtaining a fast multigrid convergence. Our comparison is based on how fast a two grid iteration converges using the two strategies.

To carry out the comparison, we construct the following two grid iteration matrix and calculate its spectral radius

$$
G_{\mathrm{D}}^{T G}=\left(I-I_{2 h}^{h}\left(A_{\mathrm{D}}^{2 h}\right)^{-1} I_{h}^{2 h} A_{\mathrm{D}}^{h}\right) G_{\mathrm{D}}^{P},
$$

where the prolongation operator $I_{2 h}^{h}$ is the linear interpolation whose matrix form is given by

$$
I_{2 h}^{h}=\frac{1}{2}\left[\begin{array}{llllll}
1 & & & & & \\
2 & & & & & \\
1 & 1 & & & \\
2 & & & \\
& 1 & 1 & & \\
& 2 & \ddots & \\
& & 1 & \ddots & \\
& & & & 1 \\
& & & & 2 \\
& & & & 1
\end{array}\right] \in \mathbb{R}^{(N-2) \times(N-3) / 2}
$$


and the restriction matrix $I_{h}^{2 h}$ is the transpose of the prolongation matrix

$$
I_{h}^{2 h}=\left(I_{2 h}^{h}\right)^{T} .
$$

The coarse grid matrix $A_{\mathrm{D}}^{2 h}$ is defined by Galerkin projection

$$
A_{\mathrm{D}}^{2 h}=I_{h}^{2 h} A_{\mathrm{D}}^{h} I_{2 h}^{h} .
$$

Because the kernel function can be resolved on every level by the underlying grid, it is certainly possible to use rediscretization to obtain $A_{\mathrm{D}}^{2 h}$. However, the Nyström method requires $\delta \geq h$ for all levels. The practical choice in the peridynamics community is $\delta=3 h$ or $4 h$, which allows only three levels at most in the hierarchy. Therefore, we decided not to use rediscretization for practical purposes. In addition, peridynamics is usually implemented as a meshless method because it aims to capture dynamic fracture. It would be ideal to construct an algebraic method with minimal reliance on the mesh. A fully algebraic method is one of our future research avenues.

In Table 1 , we report spectral radius of the matrix $G_{\mathrm{D}}^{T G}$ for varying mesh size $h=2^{l}, l=3, \ldots, 9$. when $\delta=2^{-2}$. Although as $h \rightarrow 0$, the convergence rates for the two strategies seem equivalent, for large $h$ convergence for the second strategy is faster. Overall, the second strategy seems more effective than the first one. When $h$ becomes smaller, the number of eigenvalues accumulating around zero increases, and as a result, the performance of the two methods becomes similar and they both improve.

We have implemented a multilevel hierarchy using 5 levels with weighted Jacobi using parameters $\omega=1-\frac{h_{l}}{2 \delta}$ in Table 2. When $l=2$, weighted Jacobi is equivalent to Picard's iteration. We have observed convergence indicating that the smoothers are effective for more than two levels as well.

The operators $\mathcal{T}_{\mathrm{p}}, \mathcal{T}_{\mathrm{a}}, \mathcal{T}_{\mathrm{N}}$, and $\mathcal{T}_{\mathrm{D}}$ are all compact. Hence, their discretized forms have eigenvalues that cluster at 0 for large enough $R$ (e.g., $R \geq 4$ ); see Figure 6 for the operator $\mathcal{T}_{\text {a. }}$. For small $R$ values, we do not observe this clustering property. For computational feasibility, practical $R$ values are chosen small, typically $R=3,4$, in peridynamic applications. For such practical values of $R$, the discretized operators in the multilevel hierarchy seem far from being collectively compact.

Collective compactness is a desirable property to construct an abstract general purpose framework for the convergence analysis of the multigrid method of the second kind. We do not report a convergence proof of multigrid in this paper. However, the convergence of multigrid has been established in the work of Kaya ${ }^{24}$ utilizing our operators. The approach taken in ${ }^{24}$ to convergence proof is direct because the eigenvalues of the governing operator are explicitly available. Hence, it seems that collective compactness property is not essential for the convergence proof in our case. Furthermore, we report in the work of Kaya ${ }^{24}$ that the spectral radius of the iteration matrix of the two-grid is bounded by $\frac{1}{\sqrt{2} R}$. This indicates that as $R$ gets larger (i.e., the case of increased clustering), the convergence improves and we have convergence even for $R=1$.

In the presence of collective compactness, a Krylov iteration has been shown to be an effective smoother. ${ }^{25,26}$ Based on this finding, a Krylov iteration has a potential to be an effective smoother in our case as well. Especially, for large $R$, it

TABLE 1 Spectral radius of the two grid iteration matrix $G_{\mathrm{D}}^{T G}$ for the two strategies for varying $h$ when $\delta=2^{-2}$

$\begin{array}{lcc} & \rho\left(G_{\mathrm{D}}^{T G}\right)(\text { the first strategy) } & \rho\left(G_{\mathrm{D}}^{T G}\right) \text { (the second strategy) } \\ h=2^{-2} & 0.5000 & 0.3951 \\ h=2^{-3} & 0.1727 & 0.1423 \\ h=2^{-4} & 0.1083 & 0.1054 \\ h=2^{-5} & 0.0603 & 0.0597 \\ h=2^{-6} & 0.0310 & 0.0309 \\ h=2^{-7} & 0.0156 & 0.0156 \\ h=2^{-8} & 0.0078 & 0.0078 \\ h=2^{-9} & 0.0039 & 0.0039 \\ h=2^{-10} & 0.0020 & 0.0020\end{array}$

TABLE 2 Number of iterations for convergence with fixed $\delta, \delta=2^{-4}$ and varying $h$. The exact solution $u(x)=\sin (\pi x)$ is used to compute the right-hand side

$\begin{array}{lccccccc}\boldsymbol{h}_{\boldsymbol{l}} & \mathbf{2}^{\mathbf{- 4}} & \mathbf{2}^{\mathbf{- 5}} & \mathbf{2}^{\mathbf{- 6}} & \mathbf{2}^{\mathbf{- 7}} & \mathbf{2}^{\mathbf{- 8}} & \mathbf{2}^{\mathbf{- 9}} & \mathbf{2}^{\mathbf{- 1 0}} \\ l=2 & 3 & 2 & 2 & 2 & 1 & 1 & 1 \\ l=3 & 10 & 3 & 2 & 2 & 2 & 1 & 1 \\ l=4 & 20 & 6 & 3 & 2 & 2 & 2 & 2 \\ l=5 & 56 & 13 & 4 & 5 & 4 & 3 & 2\end{array}$


seems worthwhile to investigate Krylov-based smoothers, which may be a future research direction. We also observe the weighted Jacobi becomes more effective as $R$ becomes large; see Table 2 . For small $R$, we expect iteration counts similar to ours when a Krylov-based smoother is used. Even in the absence of collective compactness, the weighted Jacobi is able to damp most oscillatory modes, which seems to play an important role in convergence. In addition, we observe a clustering behavior of eigenvalues that indicates that rather than high wavenumbers, middle wavenumbers cluster at 0 ; see Figure 6. Because Picard's iteration aims to damp the most oscillatory mode and the fact that the middle mode is the most oscillatory, for large $R$, damping the most oscillatory mode leads to damping a large number of oscillatory modes.

Remark 1. According to our ordering of the wavenumber, the eigenvalues of the discretized $\mathcal{T}_{\mathrm{BC}}$ with middle wavenumbers cluster near zero; see Figure 6. However, with reordering of wavenumbers, we can easily translate the clustering to high wavenumbers. For instance, for the case of $N=31$, the mapping

$$
\phi(k)= \begin{cases}1, & k=1, \\ 2 k-1, & k=2, \ldots 16, \\ 2(32-k), & k=17, \ldots 31,\end{cases}
$$

gives a new ordering of the wavenumbers such that the first and second halves of the eigenvectors correspond to smooth and oscillatory modes, respectively. Hence, the clustering near zero occurs when wavenumbers are high.
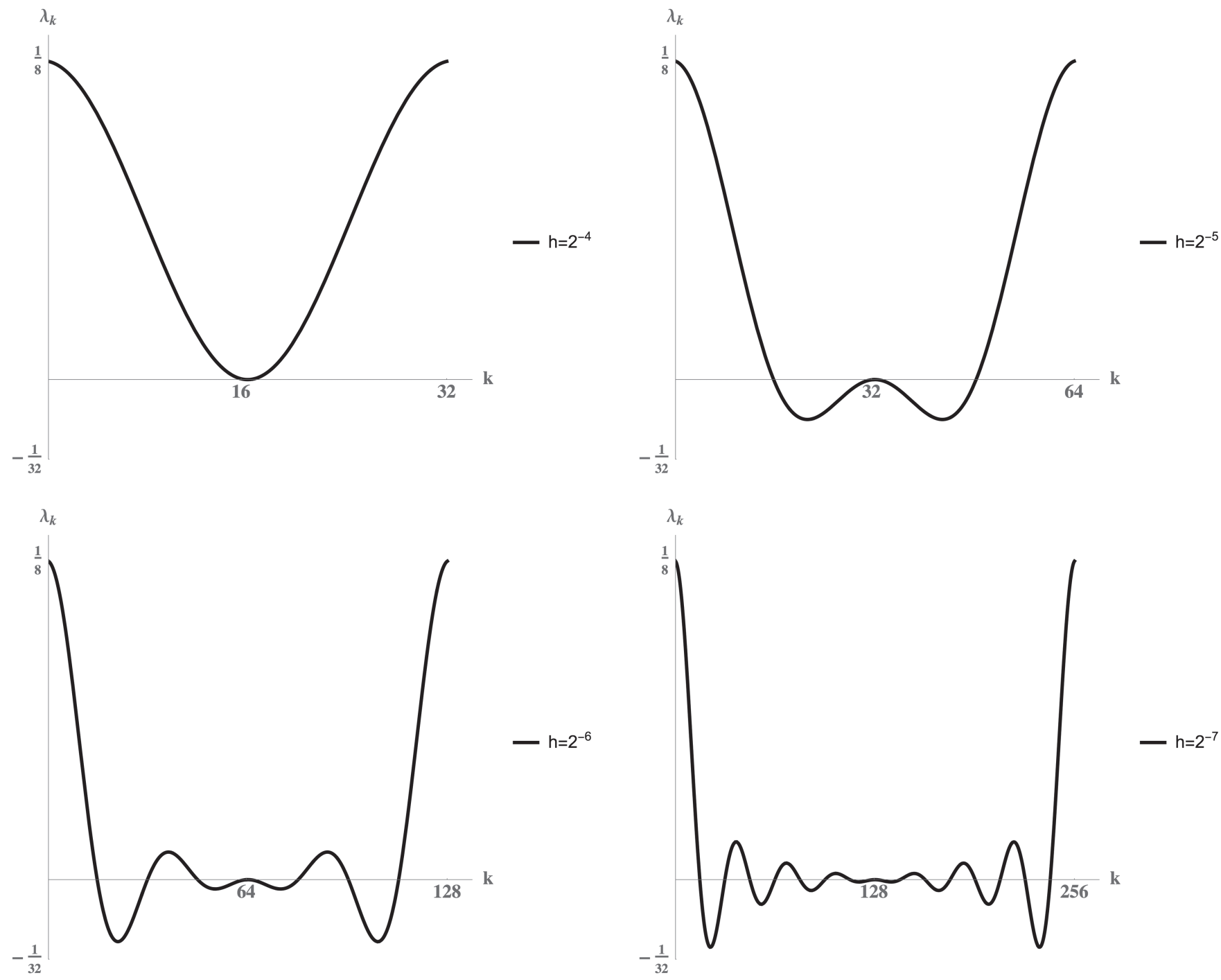

FIGURE 6 Eigenvalues of discretized $\mathcal{T}_{\mathrm{a}}$ with $h=2^{-4}, 2^{-5}, 2^{-6}, 2^{-7}$ using $\delta=2^{-4}$ 


\section{DISCUSSION ON THE PICARD ITERATION}

The Picard iteration has been used as a smoother for the multigrid of the second kind. ${ }^{20,22}$ The Fredholm second kind operator has a smoothing property and that is why the Picard iteration has been chosen as a smoother. However, to the best of our knowledge, there is no study that sheds light on its internal mechanism.

To better understand the Picard iteration, we pose the following questions:

- Does it work for all kinds of Fredholm second kind problems?

- Does the smoothing property of the Picard iteration stem from the nature of the problem under consideration?

In Section 4, we observed that it focuses on the most oscillatory mode and aims to damp it rapidly. To better understand internal mechanism of the Picard iteration, we generalize our nonlocal antiperiodic problem by allowing $c$ to be an arbitrary constant. We consider the following academic problem:

$$
c u(x)-\mathcal{T}_{\mathrm{a}} u(x)=f(x),
$$

where $\mathcal{T}_{a}$ is given in (5). Here, we assume that the constant $c>0$ and it is not an eigenvalue of $\mathcal{T}_{\mathrm{a}}$. This implies that $\mathcal{M}_{a}$ has a bounded inverse; hence, the equation (33) has a unique solution.

We apply the same discretization and algebraic operations to get the symmetric matrix. The Picard iteration for the discrete equation is defined by

$$
\mathbf{v}^{1}=\frac{1}{c} \mathcal{T}_{\mathrm{a}}^{h} \mathbf{v}^{0}+\frac{1}{c} \mathbf{f}
$$

where $\mathcal{T}_{\mathrm{a}}^{h}$ is the discrete version of $\mathcal{T}_{\mathrm{a}}$

Let $\lambda_{m o}\left(\mathcal{T}_{a}^{h}\right)$ denote the eigenvalue corresponding to the most oscillatory mode of $\mathcal{T}_{a}^{h}$. The smoothing property of the Picard iteration depends on the ratio $\frac{\lambda_{m o}\left(\mathcal{T}_{a}^{h}\right)}{c}$. When this ratio is zero or close to zero, then the Picard iteration is a perfect smoother. However, when the absolute value of the ratio is larger than 1, the Picard iteration fails to be a smoother.

We know that $\lambda_{m o}\left(\mathcal{T}_{a}^{h}\right) \neq 0$. Now, we test the Picard iteration for $c=2 \delta$ and $c=\frac{\delta^{4}}{10}$ where $\delta=0.25$. Note that for these choices, the operator satisfies the Fredholm alternative and, hence, has a unique solution. However, the operators corresponding to these cases are ill-posed and the ill-posedness is more severe for the case of $c=\frac{\delta^{4}}{10}$.

We choose the initial iteration

$$
\mathbf{v}_{i}^{0}=\cos (i 31 \pi / N)+\cos (i 5 \pi / N), \quad i=1, \ldots, N-1,
$$

with $N=33$. We set $f=0$, so that $\mathbf{e}^{k}=-\mathbf{v}^{k}$. We show the approximations after one sweep in Figure 7. For $c=2 \delta$, $\frac{\lambda_{m o}\left(\mathcal{T}_{a}^{h}\right)}{c} \doteq-0.0024$ and for $c=\frac{\delta^{4}}{10}, \frac{\lambda_{m o}\left(\mathcal{T}_{a}^{h}\right)}{c} \doteq-3.008$. This means that the oscillations in the right graph in Figure 7 cannot be damped by the Picard iteration. In summary, our academic example indicates that the smoothing property of the Picard iteration depends on the problem considered. Hence, the Picard iteration does not necessarily generate the expected smoothing for all Fredholm second kind problems.

FIGURE 7 Approximations after one sweep with the Picard iteration for $c=2 \delta$ (left) and for $c=\frac{\delta^{4}}{10}$ (right)
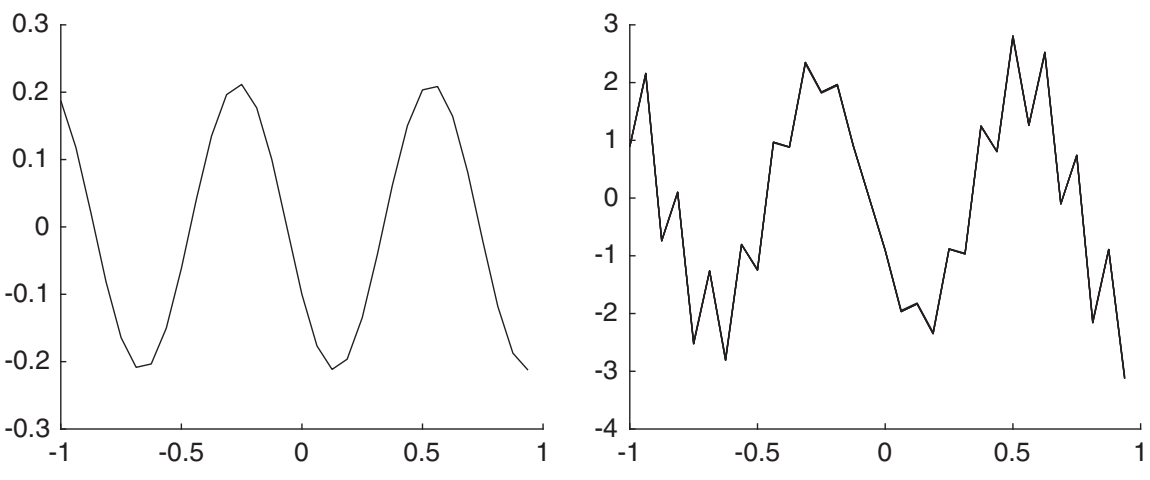


\section{7 | CONCLUSION}

We studied smoothers for the multigrid method of the second kind. We provided the eigenvalues of the resulting matrices in terms of $\delta$ and $h$. We presented a spectral analysis for the iteration matrix of the weighted-Jacobi method. We proposed two strategies. The first one focuses on the most oscillatory mode and aims to damp it effectively. For the first strategy, we found a parameter suitable for which we showed that weighted-Jacobi relaxation is equivalent to the Picard iteration.

The second strategy focuses on the oscillatory modes and aims to damp them as quickly as possible, simultaneously. When we compared the two strategies, the second strategy outperforms the first one. However, the strategies become equivalent when the mesh size goes to 0 . We shed some light on internal mechanism of the Picard iteration. We provided an example where the Picard iteration cannot be used as a smoother. To the best of our knowledge, a guiding spectral analysis is not available for smoothers of the multigrid of the second kind. We filled this gap in the literature.

\section{ACKNOWLEDGEMENTS}

Burak Aksoylu's research was sponsored by the Army Research Laboratory and was accomplished under Cooperative Agreement W911NF-18-2-0090. The views and conclusions contained in this document are those of the authors and should not be interpreted as representing the official policies, either expressed or implied, of the Army Research Laboratory or the US government. The US government is authorized to reproduce and distribute reprints for government purposes notwithstanding any copyright notation herein.

\section{CONFLICT OF INTEREST}

The authors declare no conflict of interest.

\section{ORCID}

Burak Aksoylu (D) https://orcid.org/0000-0002-7244-3340

Adem Kaya (iD) https://orcid.org/0000-0001-5838-3284

\section{REFERENCES}

1. Atkinson K, Han W. Theoretical numerical analysis. Berlin, Germany: Springer Verlag; 2009.

2. Sloan IH. Improvement by iteration for compact operator equations. Math Comput. 1976;30(136):758-764.

3. Wolff H. Multi-grid techniek voor het oplossen van Fredholm-integraalvergelijkingen van de tweede soort. Report NN 19/79. Amsterdam, The Netherlands: Mathematisch Centrum; 1979.

4. Hemker PW, Schippers H. Multiple grid methods for the solution of Fredholm integral equations of the second kind. Math Comput. 1981;36:215-232.

5. Schippers H. Multi-grid techniques for the solution of Fredholm integral equations of the second kind. In: te Riele HJJ, editor. Colloquium numerical treatment of integral equations. Amsterdam, The Netherlands: Mathematisch Centrum, 1979; p. 29-46. Mathematisch Centrum Amsterdam: MC syllabus, No. 41.

6. Hackbusch W. Die schnelle Auflösung der Fredholmschen Integralgleichung zweiter Art. Beiträge Numer Math. 1981;9:47-62.

7. Aksoylu B, Beyer HR, Celiker F. Application and implementation of incorporating local boundary conditions into nonlocal problems. Numer Funct Anal Optim. 2017;38(9):1077-1114.

8. Aksoylu B, Beyer HR, Celiker F. Theoretical foundations of incorporating local boundary conditions into nonlocal problems. Rep Math Phys. 2017;80(1):39-71.

9. Aksoylu B, Celiker F. Nonlocal problems with local Dirichlet and Neumann boundary conditions. J Mech Mater Struct. 2017;12(4):425-437.

10. Silling SA. Reformulation of elasticity theory for discontinuities and long-range forces. J Mech Phys Solids. 2000;48:175-209.

11. Kalthoff JF, Winkler S. Failure mode transition at high rates of shear loading. In: Chiem CY, Kunze HD, Meyer LW, editors. Impact loading and dynamic behavior of materials. Vol. 1. Berlin, Germany: DGM Informationsgesellschaft Verlag, 1988; p. 185-195.

12. Silling SA. Dynamic fracture modeling with a meshfree peridynamic code. In: Bathe KJ, editor. Computational fluid and solid mechanics. Oxford, UK: Elsevier, 2003.

13. Kilic B, Madenci E. Prediction of crack paths in a quenched glass plate by using peridynamic theory. Int J Fract. 2009;156:165-177.

14. Oterkus E, Madenci E. Peridynamic analysis of fiber reinforced composite materials. J Mech Mater Struct. 2012;7:45-84.

15. Silling SA, Bobaru F. Peridynamic modeling of membranes and fibers. Int J Non Linear Mech. 2005;40:395-409.

16. Silling SA, Bobaru F. Peridynamic 3D problems of nanofiber networks and carbon nanotube-reinforced composites. In: Materials and design: Proceedings of Numiform. Melville, NY: American Institute of Physics, 2004; p. 1565-1570. 
17. Aksoylu B, Celiker F, Kilicer O. Nonlocal problems with local boundary conditions in higher dimensions. Adv Comp Math. 2019;45(1):453-492.

18. Aksoylu B, Celiker F, Kilicer O. Nonlocal operators with local boundary conditions: An overview. In: Voyiadjis GZ, editor. Handbook of nonlocal continuum mechanics for materials and structures. Cham, Switzerland: Springer International Publishing, 2019; p. 1293-1330.

19. Aksoylu B, Kaya A. Conditioning and error analysis of nonlocal problems with local boundary conditions. J Comput Appl Math. 2018;335:1-19.

20. Hackbusch W. Multi-grid methods and applications. Vol. 4. Berlin, Germany: Springer-Verlag; 1985.

21. Briggs WL, Henson VE, McCormick SF. A multigrid tutorial. 2nd ed. Philadelphia, PA: SIAM; 2000.

22. Hackbusch W. Iterative solution of large sparse systems of equations. 2nd ed. Springer; 2015. Applied mathematical sciences, No. 95.

23. Trottenberg U, Oosterlee CW, Schuller A. Multigrid. Cambridge, MA: Academic Press; 2001.

24. Kaya A. Multigrid convergence analysis for nonlocal equations. 2019. Under review.

25. Lee B. A novel multigrid method for Sn discretizations of the mono- energetic Boltzmann transport equation in the optically thick and thin regimes with anisotropic scattering, part I. SIAM J Sci Comput. 2010;31:4744-4773.

26. Lee B. A multigrid framework for $S_{n}$ discretizations of the Boltzmann transport equation. SIAM J Sci Comput. 2012;34:A2018-A2047.

How to cite this article: Aksoylu B, Kaya A. On smoothers for multigrid of the second kind. Numer Linear Algebra Appl. 2019;26:e2267. https://doi.org/10.1002/nla.2267 